\title{
Cutaneous Polyarteritis Nodosa
}

National Cancer Institute

\section{Source}

National Cancer Institute. Cutaneous Polyarteritis Nodosa. NCI Thesaurus. Code C117295.

A form of vasculitis that involves small to medium size arteries of the dermis and subcutaneous tissue. The disorder manifests with tender subcutaneous nodules, livedo reticularis, cutaneous ulcers and necrosis; while internal organ involvement is typically spared, extra-cutaneous symptoms may be present. 